\title{
Master-Slave-Type Gait Training System for Hip Movement Disorders
}

\author{
Tasuku Miyoshi, ${ }^{1,2 *}$ Kenta Asaishi, ${ }^{1}$ Taishu Nakamura, ${ }^{1}$ and Motoki Takagi ${ }^{3}$ \\ ${ }^{1}$ Department of Mechanical Engineering, Faculty of Engineering, Iwate University \\ ${ }^{2}$ Soft-Path Engineering Research Center (SPERC), Faculty of Engineering, Iwate University \\ ${ }^{3}$ Organization of Revitalization for Sanriku-region, Iwate University
}

(Received October 22, 2015; accepted January 28, 2016)

Keywords: pneumatic artificial muscle (PAM), empirical model of the PAM, inertial measurement unit, symmetric gait patterns, simulated electromyographic activities

Robotic devices intended to assist patients recovering from hip movement disorders face two major problems, namely, difficulty in (1) enhancing self-efforts in gait training by only using a robotic device and (2) reproducing a patient's own coordinated motion during gait training. To solve these problems, the authors have developed a gait training device based on the master-slave system. This device treats the user's healthy limbs as the "master" and the paretic limbs for which the user wishes to achieve normal gait motions as the "slave". An inertial measurement unit is used to detect hip joint angular displacements and the time it takes for each displacement to occur; the hip joint angular displacement on the slave side is controlled by a proportional-integral-derivative (PID) controller. Six healthy persons were equipped with the device and asked to walk along a walkway so that their gait motion and the electromyographic activities in their lower limb muscles could be evaluated. The results of that experiment indicate that gait motion was preserved in the simulated paresis in the subjects' hip muscles, which suggests that the proposed master-slavetype gait training device can enhance users' efforts, and that the PID controller controlling angular displacements of the hip joint is able to reproduce natural gait motions.

\section{Introduction}

Strokes are widely known to be one of the leading causes of adult movement disabilities..$^{(1,2)}$ In most cases of poststroke hemiparesis, patients suffer from decreased walking speeds and less symmetrical gait patterns. Note that the term "gait motion" refers to the repetitive pattern of the two lower limb segments originating from a person's hip joint; the symmetrical pattern is thus determined by the timing and coordination of the left and right legs as they support the other and swing. Although gait recovery is usually achieved within 12 weeks of a stroke, the recovered gait is often incomplete and characterized by an asymmetric pattern. ${ }^{(3)}$ Recent studies have demonstrated that the reciprocal hip flexion/extension relationship between the patterns of right and left hip joint angular displacements plays an important role in enhancing the activation of the spinal locomotor circuit. $^{(4,5)}$ Thus, patterns of hip joint displacement during walking are one of the most effective indications of gait rehabilitation.

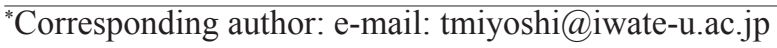


For patients with movement disabilities, voluntarily regaining one's natural gait is difficult. In a previous study, to enhance the training effects of poststroke physical therapy, patients were instructed to move their paralyzed limbs by mirroring the natural movements of their healthy limbs. ${ }^{(6)}$ The same effects that were thus obtained with stroke patients were then also achieved for patients with chronic spinal cord injuries. ${ }^{(7)}$ On the basis of these past studies, we consider that gait rehabilitation can be achieved when (1) the symmetrical gait patterns in the hip joint are preserved and (2) voluntary self-efforts are encouraged to enhance the gait training effects of physical therapy ${ }^{(8)}$ because robotic devices provide transitive exercises. ${ }^{(9-11)}$ On the basis of these two points, we propose a novel gait training device for use with the hip joint, which is based on master-slave systems. In this study, we aim to develop a device capable of aiding patients to regain bilateral and symmetrical patterns in their hip joints through enhanced self-effort and then assess this newly developed device in terms of its effectiveness. Six healthy subjects participated in the assessments, which examined the subjects' kinematic, kinetic, and simulated electromyographic (EMG) activities.

\section{Materials and Methods}

\subsection{Concepts of the gait training device based on the master-slave system}

Our device was designed on the basis of the following assumptions about the patients using it: patients should be no taller than $180 \mathrm{~cm}$, weigh no more than $80 \mathrm{~kg}$, suffer from left side hemiparesis, be able to walk independently with the use of either a cane or Lofstrand crutch, and exhibit asymmetrical gait motions in their hip joint angular displacements.

Ideally, a patient's right and left legs should be symmetrical and the reciprocating differences between the two should be approximately $50 \%$ of one gait cycle. Therefore, we constructed a master-slave-type gait training system so that feedback could be obtained from the healthy side; this is carried out by setting the master signal to be the changing pattern of the healthy leg's joint angle while the patient walks and setting the slave signal (on the paretic side) to have reciprocating differences of $50 \%$ of the master signal's gait cycle.

\subsection{Sensors system}

To measure the hip flexion/extension joint angular displacements of the healthy side's natural gait, inertial measurement units (IMU, GY-521, Shenzhen Taida Century Technology Co., Ltd.) used the master signal's acquisition. The right hip joint angular displacement of the healthy side was measured as the master signal $\left(\theta_{\mathrm{mst}}\right)$. The specifications of the master device are as follow: $87 \mathrm{~mm}$ wide, $29 \mathrm{~mm}$ high, $35 \mathrm{~mm}$ deep, $50 \mathrm{~g}$ in weight, and $50 \mathrm{~mA}$ of current consumption. The master device was attached to subject's right thigh using a Velcro strap, and the signals were recorded via I2C communication to a microcomputer board (Arduino Pro Mini, Arduino Inc.) and then via serial communication to the PC for slave device control. Detecting hip joint angular displacements on the slave side, a potentiometer (RV16YN15S, Tokyo Cosmos Electric Co., Ltd.) was attached to the hip axis to obtain feedback signals $\left(\theta_{\text {out }}\right)$. All of the control signals were sampled at $100 \mathrm{~Hz}$. It should be noted that the IMU was not used to measure the hip joint angle of the slave side, since the communication cable of IMU device would inhibit the attachment/detachment of the slave device. 


\subsection{Slave side system configuration}

\subsubsection{Empirical model of the pneumatic artificial muscle (PAM)}

To reduce the weight of the device, we used PAMs (Kanda Tsushin Kogyo Co., Ltd.). A pair of PAMs (each of which had a 1.5 inch diameter, weighed $150 \mathrm{~g}$, and had a natural length $\left(L_{\text {nat }}\right)$ of 430 $\mathrm{mm}$ ) was equipped around the hip joint to achieve the same hip range of motion as that achieved by a pair of agonistic-antagonistic muscles. By using PAM in the master-slave gait training system, we were able to gain insight into the derivation of the empirical model because the relationships among changes in the length of PAM, pulling loads, and supplied air pressure demonstrate nonlinear characteristics. ${ }^{(12,13)}$ The step responses of changes in the PAM's length were recorded as a function of supplied air pressure and additional loads attached to the PAM. The supplied compressed air ranged from 0.1 to $0.5 \mathrm{MPa}$ (0.1 MPa steps) and was supplied via an electropneumatic regulator (ITV 2000 series, SMC Corporation); attached loads were set at 5, 10, and $15 \mathrm{~kg}$. Changes in the PAM's length were recorded using a linear potentiometer (LP-250FJ, Midori Precisions Co., Ltd.). Each condition was carried out 5 times, and all analog signals were synchronized and recorded through an A/D converter (PCI-3521, Interface Corporation) for offline analysis. We constructed a threedimensional (3D) plot of the contraction ratio of the PAM ( $z$-axis) as a function of the pulling loads and supplied air pressure. To understand the 3D plot, the PAM's contraction ratio was obtained from regression as

$$
z=a_{0}+a_{1} x+a_{2} p+a_{3} x^{2}+a_{4} p^{2}+a_{5} x p
$$

where $z$ is the contraction ratio (percentage of the natural length of the PAM), $x$ is the pulling load $(\mathrm{kg}), p$ is the supplied air pressure (MPa), and $a_{0}, a_{1}, a_{2}, a_{3}, a_{4}$, and $a_{5}$ are the coefficients, which are calculated by the least squares method.

\subsubsection{Acquiring the slave side hip flexion/extension joint angular displacements}

The slave device was constructed on the basis of the trunk-obtained hip orthosis (Fig. 1). The hip joint's range of motion was set at $30^{\circ}$ for flexion and $10^{\circ}$ for extension to achieve natural and symmetrical gait motion in healthy subjects. The slave device consisted of two main links $\left(L_{0}=\right.$ 220 and $\left.L_{1}=275\right)$ and five attachments $\left(L_{2}=50, L_{3}=140, L_{4}=230, L_{5}=50\right.$, and $\left.L_{6}=50 \mathrm{~mm}\right)$ with a flange whose diameter $(R)$ is $100 \mathrm{~mm}$. Since the supposed weight of the subject was $80 \mathrm{~kg}$ and the ratio of the thigh's mass against that of the body was approximately $16 \%,{ }^{(14)}$ we assumed a 15 $\mathrm{kg}$ loading and $0.3 \mathrm{~kg}$ for a pair of PAMs at a safety factor of 1.2 to generate hip motions on the slave side.

The effective angle of the slave device was uniquely determined on the basis of PAMs' lengths for flexion $\left(L_{\mathrm{f}}\right)$ and extension $\left(L_{\mathrm{e}}\right)$. Therefore, the supplied air pressure $\mathbf{P}=\left(P_{\mathrm{f}}, P_{\mathrm{e}}\right)^{\mathrm{T}}$, which was calculated using Eq. (1) and which followed the geometric constraint that corresponds to $\left(\theta_{\mathrm{mst}}\right)$, was used as a control variable for the slave device. The PAMs' flexion and extension lengths $\mathbf{L}=\left(L_{\mathrm{f}}, L_{\mathrm{e}}\right)^{\mathrm{T}}$, exertion forces $\mathbf{F}=\left(F_{\mathrm{f}}, F_{\mathrm{e}}\right)^{\mathrm{T}}$, and supplied air pressures $(\mathbf{P})$ were as follows: 


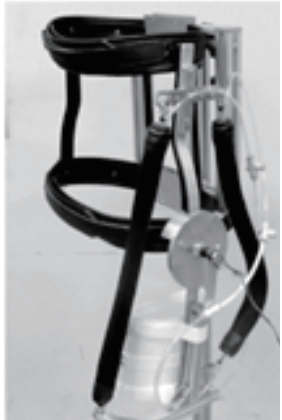

(a)

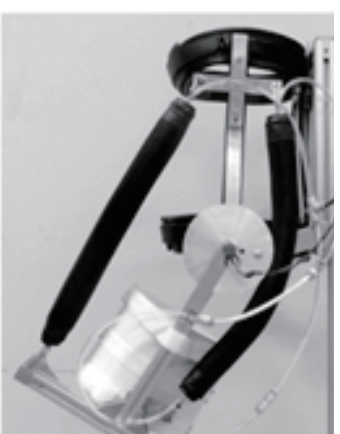

(b)

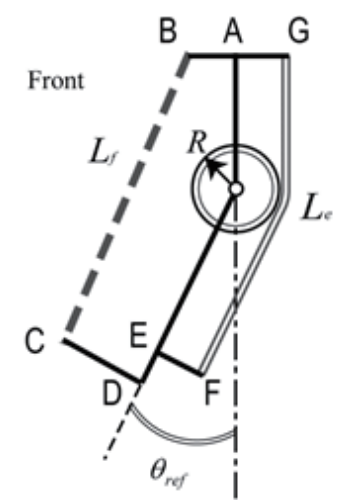

(c)

$\mathrm{OA}=L_{0}$

$\mathrm{OD}=L_{1}$

$\mathrm{AB}=L_{2}$

$\mathrm{DC}=L_{3}$

$\mathrm{OE}=L_{4}$

$\mathrm{AG}=L_{5}$

$\mathrm{EF}=L_{6}$

Fig. 1. (a) Newly developed gait training device for hip joint movement disabilities based on master-slave robotics; (b) typical hip joint flexion movement; and (c) schematic figure of the link mechanism.

$$
\begin{aligned}
& L_{\mathrm{f}}=\sqrt{\sum_{i}^{i=4} L_{i}^{2}+2 \sqrt{L_{0}^{2}+L_{2}^{2}} \sqrt{L_{1}^{2}+L_{3}^{2}} \cos \left(\theta_{\mathrm{mst}}+\tan ^{-1}\left(\frac{L_{2}}{L_{0}}\right)+\tan ^{-1}\left(\frac{L_{3}}{L_{1}}\right)\right)} \\
& L_{\mathrm{e}}=\sqrt{L_{0}^{2}+L_{4}^{2}+L_{5}^{2}+L_{6}^{2}+2 \sqrt{L_{0}^{2}+L_{5}^{2}} \sqrt{L_{4}^{2}+L_{6}^{2}} \cos \left(\theta_{\mathrm{mst}}+\tan ^{-1}\left(\frac{L_{5}}{L_{0}}\right)+\tan ^{-1}\left(\frac{L_{6}}{L_{4}}\right)\right)} \quad\left(\theta_{\mathrm{mst}}<0\right) \\
& =\sqrt{\left(L_{5}-R\right)^{2}+L_{0}^{2}}+R \theta_{\mathrm{mst}}+\sqrt{\left(L_{6}-R\right)^{2}+L_{0}^{2}} \\
& \left(\theta_{\text {mst }} \geq 0\right) \\
& F_{\mathrm{f}}=\frac{m g L_{\mathrm{f}} L_{1} \sin \theta_{\mathrm{mst}}}{\sqrt{L_{0}^{2}+L_{2}^{2}} \sqrt{L_{1}^{2}+L_{3}^{2}} \sin \left(\theta_{\mathrm{mst}}+\tan ^{-1}\left(\frac{L_{2}}{L_{0}}\right)+\tan ^{-1}\left(\frac{L_{3}}{L_{1}}\right)\right)} \\
& F_{\mathrm{e}}=\frac{m g L_{\mathrm{e}} L_{4} \sin \theta_{\mathrm{mst}}}{\sqrt{L_{0}{ }^{2}+L_{5}^{2}} \sqrt{L_{4}{ }^{2}+L_{6}{ }^{2}} \sin \left(\theta_{\mathrm{mst}}+\tan ^{-1}\left(\frac{L_{5}}{L_{0}}\right)+\tan ^{-1}\left(\frac{L_{6}}{L_{4}}\right)\right)} \\
& \mathbf{P}=\frac{-\left(a_{2}+a_{5} \mathbf{F} / g\right)+\sqrt{\left(a_{2}+a_{5} \mathbf{F} / g\right)^{2}-4 a_{4}\left(a_{3}(\mathbf{F} / g)^{2}+a_{1} \mathbf{F} / g+a_{0}-\mathbf{Z}\right)}}{2 a_{4}}
\end{aligned}
$$

where $\mathbf{Z}$ is the PAMs' contraction ratio $\left(\mathbf{Z}=\mathbf{L} / L_{\text {nat }}\right)$ and $g \mathrm{~m} / \mathrm{s}^{2}$ is gravitational acceleration. 


\subsection{Joint angular position controller based on the proportional-integral-derivative (PID) theorem}

Figure 2 shows the hip joint angular position controller for the slave side, which is based on the PID control theory. At first, acquiring the hip flexion/extension joint angular displacements on the master side $\left(\theta_{\mathrm{mst}}\right)$, the first five consecutive data points were used in the calculation, and the obtained data points throughout one gait cycle were averaged for a total of 10 gait cycles. In this study, one gait cycle was defined to last from one right heel strike to the next.

Then, the master signal $\theta_{\mathrm{mst}}$ set the bounds based on the range of motion of the slave device, and this output was used as the reference value $\left(\theta_{\text {ref }}\right)$. One such controller was constructed for each PAM, which moved the hip flexion and extension separately. The gains of each controller were as follows: proportional gains were set at $3.65 \times 10^{-3}$ for flexion and $8.00 \times 10^{-3}$ for extension; integral gains were set at 0.402 for flexion and 1.00 for extension; and differential gains were set at 2.61 $\times 10^{-6}$ for flexion and $6.67 \times 10^{-6}$ for extension. The output signal from the PID block $(\Delta \theta)$ was then converted to pressure deviations $(\Delta \mathbf{P})$ with a constant coefficient $K_{P-\boldsymbol{\theta}_{\mathrm{PID}}}=1$. Using Eq. (6), the calculated pressure $(\mathbf{P}+\Delta \mathbf{P})$ was supplied to each PAM and followed the boundary condition 0 $\mathrm{MPa} \leq(\mathbf{P}+\Delta \mathbf{P})=\mathbf{P}_{\text {out }} \leq 0.5 \mathrm{MPa}$. Finally, the slave system was able to work, the hip joint angular displacement $\left(\theta_{\text {out }}\right)$ was the output, and the loops operated repeatedly based on the master signal.

\subsection{Handling and evaluation in healthy subjects}

To assess the gait kinematic, kinetic, and EMG activities obtained using the newly developed gait training device, six healthy male subjects $(22.0 \pm 1.1$ years old, $171.0 \pm 5.9 \mathrm{~cm}$ height, $68.2 \pm$ $9.7 \mathrm{~kg}$ weight) participated in this study. The subjects had no history of orthopedic or neurological ailments or any recent injury or surgery that affected their ability to walk and/or stand upright. Each subject provided informed consent, acknowledging that the experimental procedures were approved by the ethics committee of Iwate University.

Before the trial sessions began, all the subjects were asked to practice using the gait training device for 5 min of treadmill walking so that the subjects could get used to using it. They were then asked to walk $10 \mathrm{~m}$ with the device (referred to as the "device driving condition"), and each trial was conducted 10 times per subject. To better examine the device's effects, the subjects were also asked to walk $10 \mathrm{~m}$ without the device (referred to as the "normal walking condition"), and these trials were also conducted 10 times per subject. A force platform (AccuGait, Advanced Mechanical

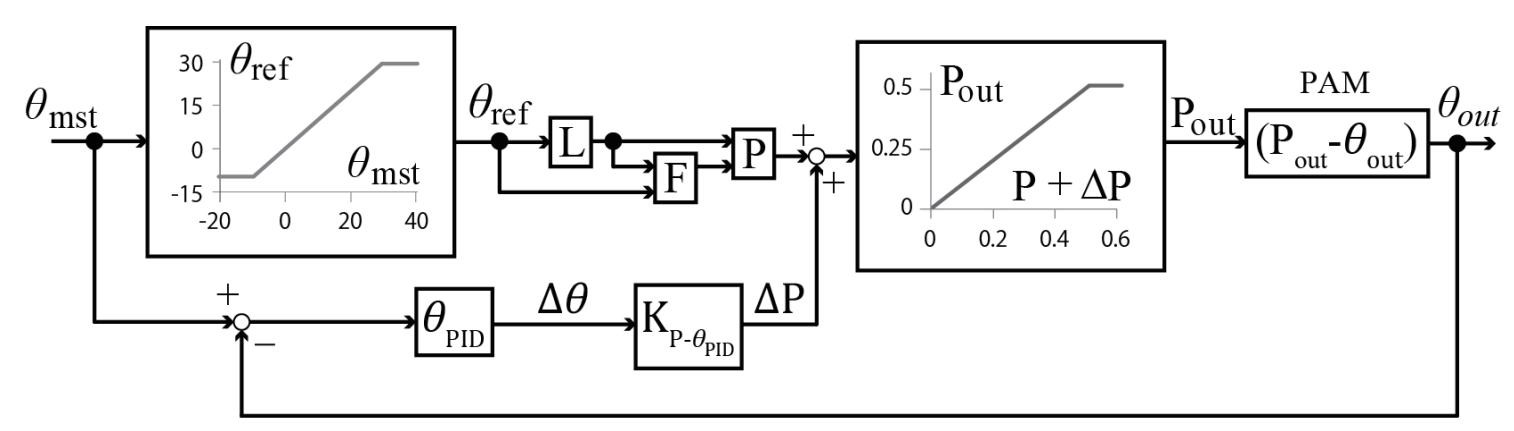

Fig. 2. Hip joint angular displacement control algorithm of the slave system based on a PID controller. 
Technology, Inc.) was set at the center of the walkway to measure the ground reaction forces and the timing of each heel strike at the fourth step.

A real-time, 3D motion analysis system (VENUS3D, Nobby Tech Ltd.) with six cameras was used to detect gait motions with 14 reflective markers attached to anatomical landmarks on both sides of each subject: the acromia, anterior superior iliac spine, greater trochanter, femoral condyles, external malleolus, and fifth metatarsophalangeal joint. All marker coordinates were sampled at $100 \mathrm{~Hz}$ and signals from the force plate were sampled at $1000 \mathrm{~Hz}$ for offline analysis. Hip, knee, and ankle angular displacements in the sagittal plane were calculated as kinematic parameters; these angles were defined as the deviation from the natural stance position. The hip flexion angle, knee flexion angle, and ankle dorsiflexion angle were defined as positive.

A body modeling system (AnyBody Modeling System, AnyBody Technology A/S) was used to calculate the following kinetic parameters: the hip, knee, and ankle joint moments in the sagittal plane, and EMG activities in the gluteus maximus (GM), rectus femoris (RF), biceps femoris longus head (BF), vastus medialis (VM), vastus lateralis (VL), medial gastrocnemius (GAS), soleus (SOL), and tibialis anterior muscle (TA) from the left side of the lower limbs. The maximum forces of the eight selected muscles were set as follows: $961.1 \pm 131.6 \mathrm{~N}$ for GM, $1263.8 \pm 173.3 \mathrm{~N}$ for RF, $2379.4 \pm 326.2 \mathrm{~N}$ for BF, 1016.7 $\pm 139.4 \mathrm{~N}$ for $\mathrm{VM}, 2582.1 \pm 354.0 \mathrm{~N}$ for $\mathrm{VL}, 3366.4 \pm 378.9$ $\mathrm{N}$ for GAS, $2416.2 \pm 272.0 \mathrm{~N}$ for SOL, and $681.0 \pm 76.7 \mathrm{~N}$ for TA. In addition, under the assumed user case (where the left side of the lower limbs was paralyzed and users could not exert any muscle force voluntarily), we simulated EMG activities with $0 \mathrm{~N}$ maximum forces in the RF and BF (referred to as the "simulated EMG condition").

The averaged profiles of the hip, knee, and ankle joint angular displacements, the joint moments, and the simulated EMG activities obtained from the trials were depicted to find any significant differences that existed between conditions. Furthermore, varying EMG activities found in one gait cycle for each muscle were examined from one gait cycle divided into 10 divisions. A oneway repeated ANOVA was applied to detect any significant differences that existed in the EMG amplitudes for each division, and multiple comparison tests were applied to detect any significant differences that existed between conditions. Significant differences in $p<0.05$ were considered acceptable.

\section{Results}

\subsection{Empirical model of the PAM}

Figure 3(a) shows the step responses of the contraction ratio as a percentage of the PAM's natural length in the working range of air pressure $(0.1-0.5 \mathrm{MPa})$. Although contraction ratios without any load increased as the input air pressure increased, the differences between other conditions were not linear, as is clearly demonstrated in Fig. 3(b). The 3D plot suggests that the PAM apparently followed nonlinear characteristics as a function of air pressure and load. This empirical model of the PAM was explicitly taken as a second-order polynomial function of air pressure, loads, and the contraction ratio as defined in Eq. (1). The coefficients are $a_{0}=-1.024, a_{1}$ $=-0.5341, a_{2}=120.7, a_{3}=0.001257, a_{4}=-151.5$, and $a_{5}=0.6162$. 


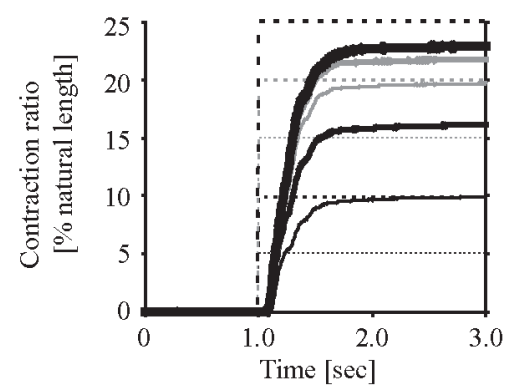

(a)

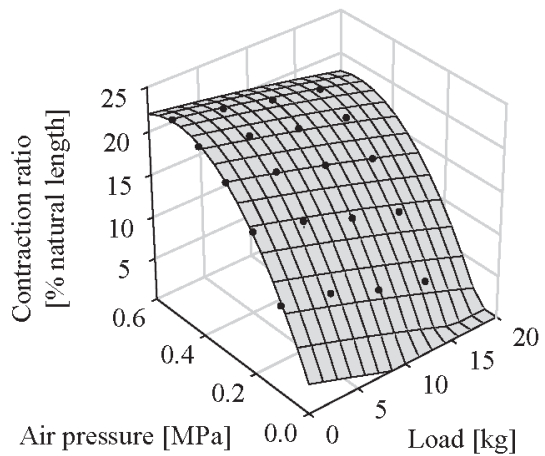

(b)

Fig. 3. (a) Typical example of the step responses of the PAM without any load and (b) an empirical model of the PAM showing the relative relationships of the contraction ratio as a function of the supplied air pressure and attached loads. In (a), a black thick line denotes $0.5 \mathrm{MPa}$ pressure; a gray thick line, $0.4 \mathrm{MPa}$; a gray thin line, 0.3 $\mathrm{MPa}$; a black medium line, $0.2 \mathrm{MPa}$; and a black thin line, $0.1 \mathrm{MPa}$.

\subsection{Kinematics and kinetics using the gait training device}

Figure 4 shows the master signal of the hip joint angular displacements measured by the IMU and the subsequent results of the 3D motion analysis. Although the movements recorded by the IMU at the peak of each cycle were slight compared with the results of the motion analysis, the cross-correlation coefficient obtained by applying the cross-correlation function between these two signals is 0.921 . It is clear from these results that the proposed device is able to provide real-time control (minus the time required for cross-correlation calculations, which is $0.063 \mathrm{~s}$ ).

Figure 5 shows the averaged profiles of (a) the joint angular displacements and (b) joint moments occurring in the sagittal plane of one subject under the normal walking condition. The patterns shown in the figure demonstrate the same tendencies. ${ }^{(15)}$ Thus, the results are reproducible and reliable enough to warrant further analysis.

Figure 6(a) shows a hip joint moment pattern that is applied to the slave device based on the master signal and is calculated using the PID controller. The figure clearly demonstrates that additional hip joint moment patterns followed hip joint angular displacements, as is also shown in Fig. 5(a): (1) the constant hip extension moment was applied from the heel-strike phase to the toeoff phase, and (2) the hip flexion moment was applied in accordance with changes in the acute hip flexion angle after the toe-off phase to the middle swing phase. Figure 6(b) shows the joint angular displacements that occurred in one subject while walking with the device. In contrast, the hip joint moment patterns obtained under the device driving condition [Fig. 6(c)] are clearly different from those obtained under the normal walking condition [Fig. 5(b)]. Although the hip joint's extension peak moment appeared at the early-to-middle stance phase during normal walking, that peak disappeared when the device was used. In addition, the hip joint's flexion peak moment appeared at the early-to-middle swing phase during normal walking, but the hip extension moment appeared at that time when the device was used. 


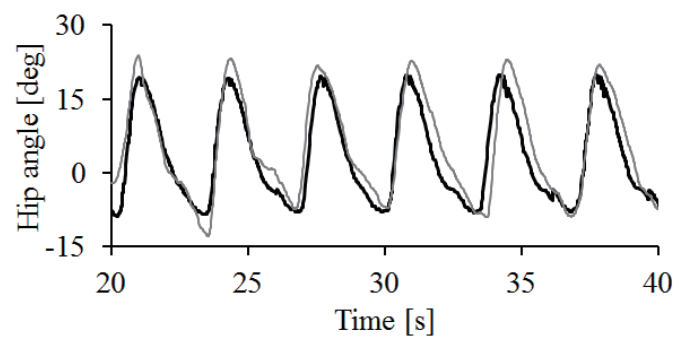

Fig. 4. Typical responses of the hip joint angular displacements; the black line denotes the master signal detected by the IMU and the gray line denotes the hip joint angular displacement calculated from 3D motion analysis.
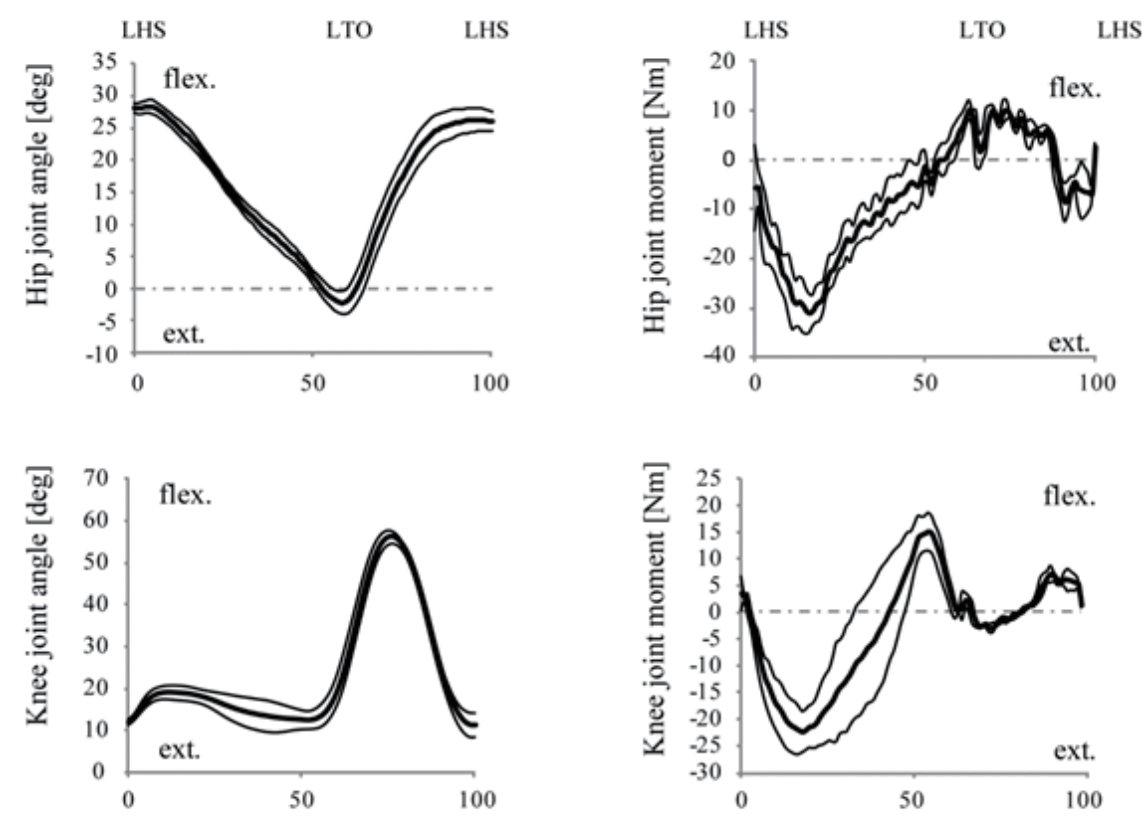

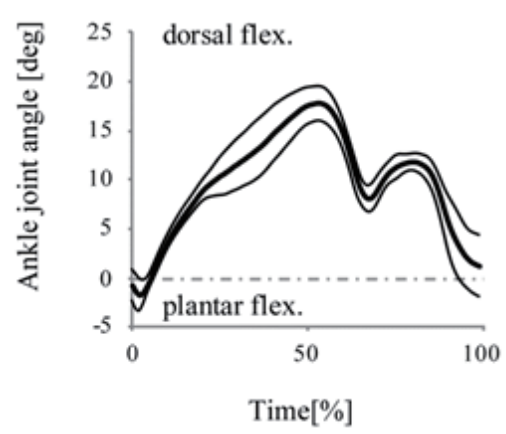

(a)

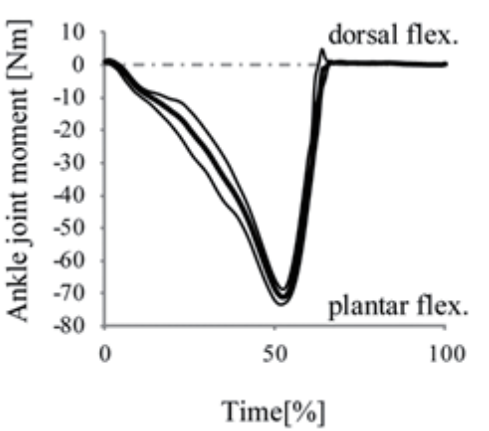

(b)

Fig. 5. (a) Typical examples of the hip (top), knee (middle), and ankle joint angular displacements (bottom) in one subject. (b) Typical examples of the hip (top), knee (middle), and ankle joint moments (bottom) in the same subject. Thick lines denote the mean value and thin lines denote the standard deviations found in one gait cycle. 


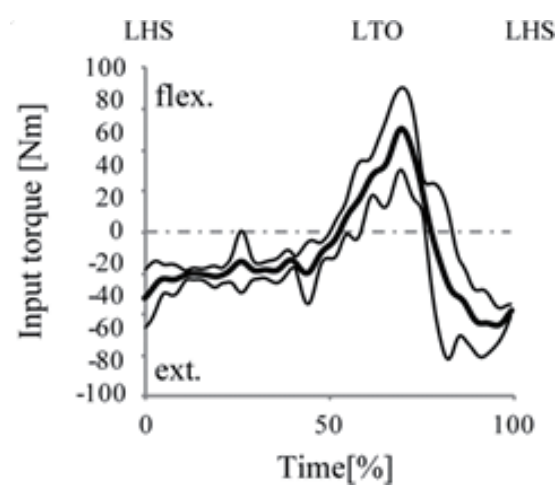

(a)
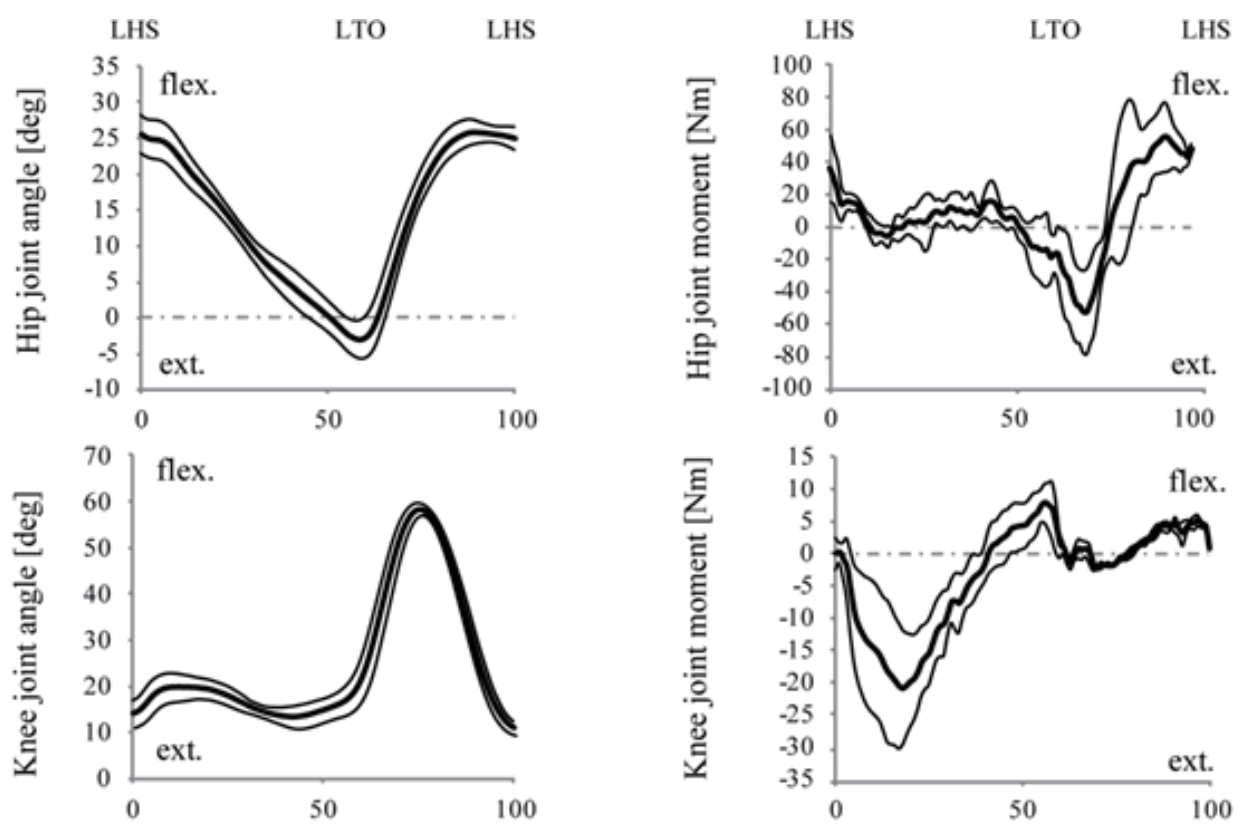

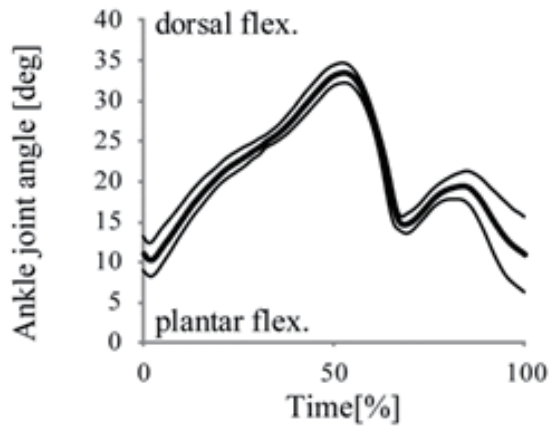

(b)

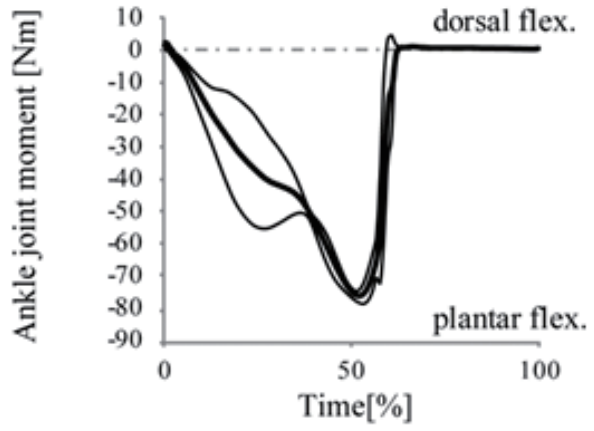

(c)

Fig. 6. (a) Input torque to drive the slave device as calculated by the hip joint angular displacement controller shown in Fig. 2. (b) Typical examples of the hip (top), knee (middle), and ankle joint angular displacements (bottom) in the same subject as that shown in Fig. 5. (c) Typical examples of the hip (top), knee (middle), and ankle joint moments (bottom) in the same subject. 


\subsection{Simulated EMG activities}

Figure 7 shows the averaged profiles of the EMG activities (a) under the normal walking condition and (b) under the device driving condition as well as (c) the simulated EMG activities under the maximum muscle force of RF when $\mathrm{BF}$ was set to $0 \mathrm{~N}$. Under the device driving condition, the activation level of the RF (i.e., the hip flexion muscle) was higher throughout the stance phase than under the normal walking condition. From the early-to-middle swing phase, when the device was used, the activation level of the hip extension muscles (i.e., the GM and BF) and knee extension muscles (i.e., the VM and VL) were higher than under the normal walking condition. In addition, while driving the device under the simulated EMG condition, the GM, VL, and VM's EMG activities were selectively enhanced compared with those that occurred without the device being used.

To clarify these tendencies, Fig. 8 shows the grand averages of all EMG activities that occurred throughout one gait cycle that had been divided into 10 divisions. There were significantly fewer EMG activities in the GM under the device driving condition than there were under both normal walking and simulated EMG conditions. In contrast, there were significantly more EMG activities in the GM, VL, and VM at the swing phase under both device driving and simulated EMG conditions than under the normal walking condition. Except for the one division for GAS that occurs at the stance phase, there were no significant differences found in ankle joint muscles (GAS, SOL, and TA) across all three conditions.

\section{Discussion}

The goal behind using a master-slave-type gait training device is to preserve the symmetrical motions of one's own gait by also enhancing the user's self-efforts. For the present study, we constructed such a device that implements three separate components: (1) an IMU to measure the hip joint angle from the healthy (i.e., master) side, (2) a hip joint angle position controller, and (3) PAMs for the slave side device. The results reported in Sec. 3 demonstrate that (1) this device can preserve the gait motion (kinematics) of a user's normal walking movements, but (2) significant differences exist between the joint moments and EMG activities (kinetics) that occur with and without the device. However, the extent to which findings from this study can be applied to patients with movement disabilities is unclear because the data is based on experiments that were limited to healthy subjects.

\subsection{Kinematic and kinetic behaviors while driving the device}

Because the hip joint angular displacement patterns shown here with the gait training device have tendencies similar to those obtained without the device [Fig. 5(a)], it is apparent that the device was able to preserve the natural gait motion of the user and that our custom designed hip joint angular position controller based on PID theorem worked sufficiently well. However, as shown in Figs. 5(a) and 6(b), there are some deviations in the hip angular displacements throughout one gait cycle. Further studies are needed to reduce these phenomenon. Recent studies have tried to implement the adaptive control theorem to the gait assist device, since these methods could modulate the feedback gains online.(16,17) 


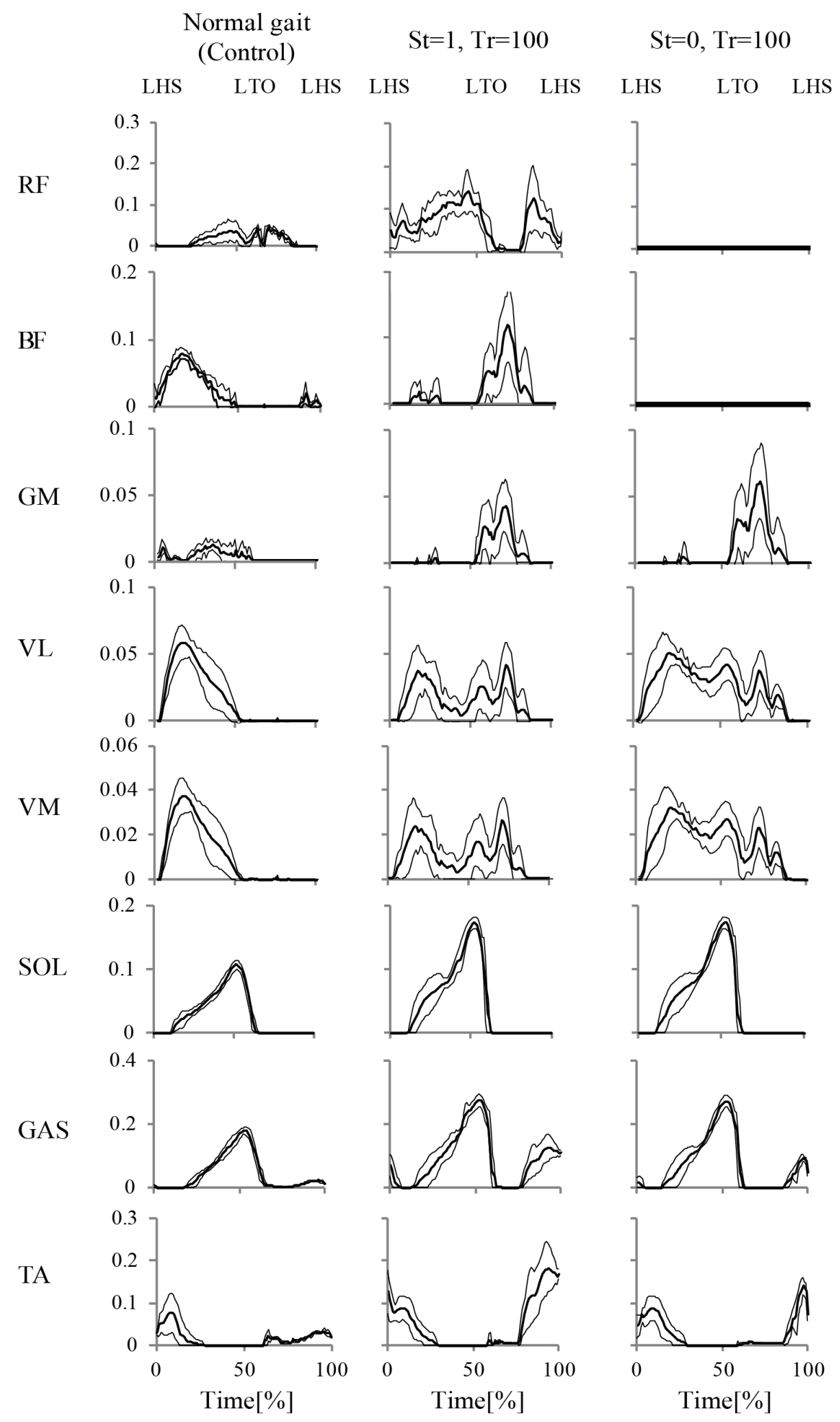

Fig. 7. Grand averaged EMG activities in the lower limbs (a) under the normal walking condition, (b) under the device driving condition, and (c) under the simulated EMG condition. Thick lines denote the mean value and thin lines denote the standard errors. 


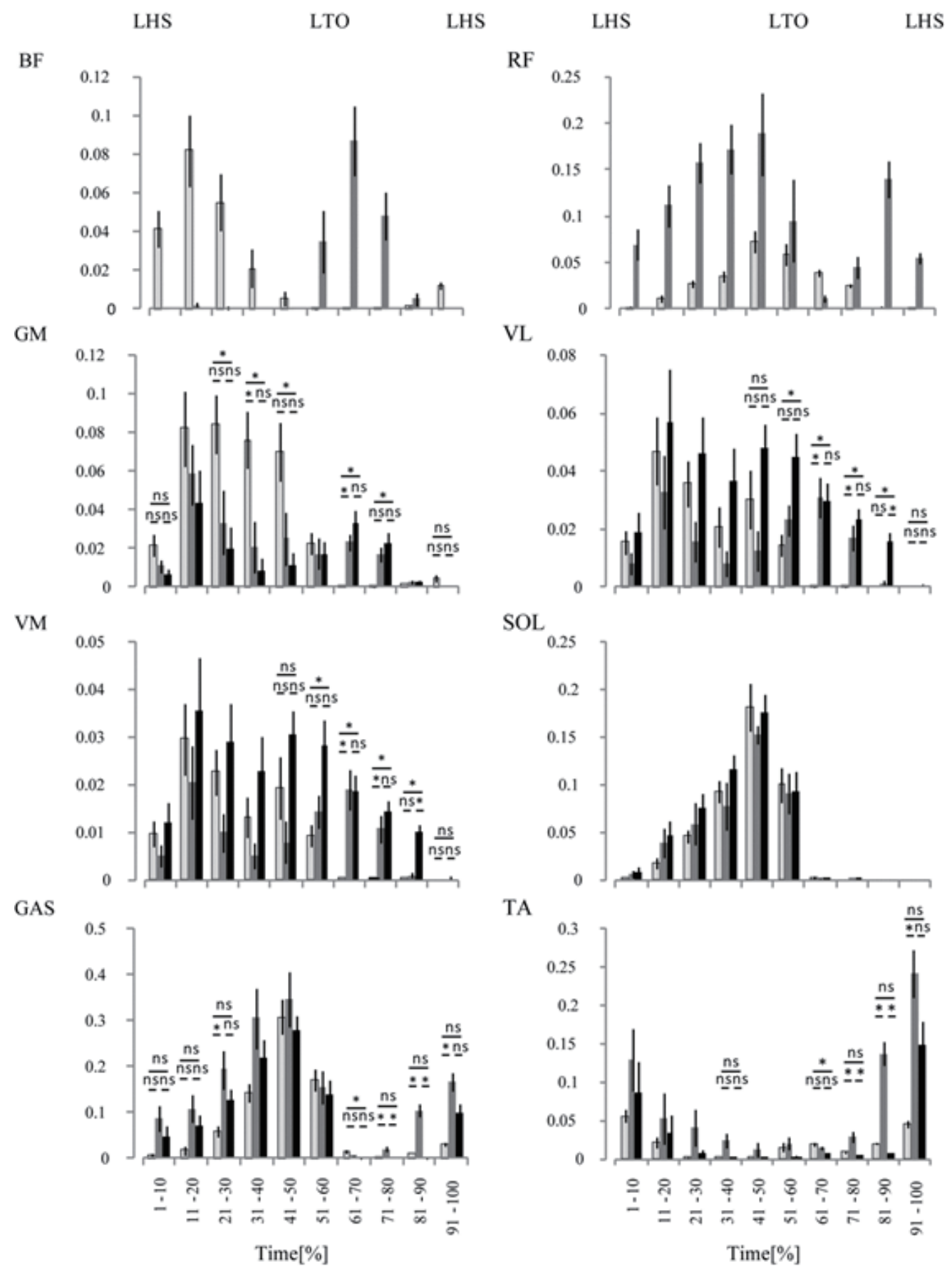

Fig. 8. EMG activities in each division. The gray solid bars denote the case under the normal walking condition; the dark gray solid bars denote the case under the device driving condition, and the black solid bars denote the case under the simulated EMG condition. Statistical differences were accepted at $p<0.05$, as shown with an asterisk (*). 
During the early-to-middle stance phase (when walking normally), the functional role of the hip joint flexion moment is to stably control the body's center of mass against the body's oscillation. ${ }^{(18)}$ In contrast, the role of the hip joint extension moment during the late stance phase (again, when walking normally) is to propel the body forward. ${ }^{(19)}$ However, the results show that the hip joint moment patterns while driving the device were quite different throughout the gait cycle from those that occurred under the normal walking condition. Thus, the results suggest that the functional role of the hip joint flexion moment while driving the device is to better stabilize the body's trunk since the device could generate that propulsive force without any self-effort from the user.

Recently, it was demonstrated that the quadriceps strength, quadriceps size, and gait parameters (i.e., stride length, step length, velocity, and cadence) were improved by isokinetic training. ${ }^{(20)}$ In this study, as shown in Fig. 6(a), the input torque profiles were not constant values and depended on the kinematic constraints. Further studies are needed to clarify the effect of the constant torque input to assist the hip joint angular displacement; kinematic dependent torque input should be suitable to preserve the gait kinematics. Therefore, it is clear that the device manages to preserve the same kinematics that appear under the normal walking condition but not the kinetics that thereby appear.

\subsection{Simulated EMG activities in lower limbs}

The EMG activities in the hip joint muscles (RF, BF, and GM) during the swing phase were significantly higher under the device driving condition than under the normal walking condition. As shown in Fig. 6(a), these muscles generate the hip flexion/extension moments in a direction opposite to that of the input joint moment occurring under the device driving condition. Thus, these results suggest that such EMG activities may inhibit the preservation of normal-walking gait kinetics when the device is used. Similarly, the EMG activities in the knee joint muscles (VL and VM) during the stance phase tended to be higher under the simulated EMG condition than under the normal walking condition. As shown in Fig. 5, the VL and VM compensate for the RF's loss of muscle force since the stance phase is also the knee extension phase and the RF, VL, and VM are the synergistic muscles for knee extension. Finally, the EMG activities in the knee joint muscles (VL and $\mathrm{VM}$ ) at the initial stance phase tended to be less under the device driving condition than they were under the normal walking condition. When we limit our results to those obtained during the initial stance $(0-10 \%)$, the activities of the knee joint extension muscles seem to contribute to the mitigation of impact force, ${ }^{(21)}$ which suggests that using the device makes it possible to reduce the impact force that occurs during the initial stance phase.

In terms of the different gait training effects that can be obtained by each to assist users in their hip joint movements, our group previously reported on the differences that existed between using a hip joint position controller that was based on hip joint angular displacements and using a hip joint moment controller that was based on the activities of the hip joint biarticular muscle group (the RF and $\mathrm{BF}$ ). ${ }^{(22)}$ However, because we have not considered the monoarticular muscle activities that occur in muscles such as the GM, VL, and VM, further research is needed before gait training devices can be widely constructed. It is interesting that the number of monoarticular muscle activities, GM, VL, and VM, increased during the swing phase under the simulated EMG condition. Historically, monoarticular muscle activities were considered to affect only one joint moment, i.e., the so-called "mechanical pulling direction". ${ }^{(23)}$ Recent studies, however, have demonstrated that monoarticular muscle activities affect the joint moment regulation of both adjacent joints, i.e., the 
so-called "preferred direction". (24-26) According to preferred direction analysis, ${ }^{(21)}$ GM, VM, and VL activations are synergistically generated at the hip extension and knee extension moments, which suggests that the increased activities of those muscles during the swing phase under both device driving and simulated EMG conditions occur to generate the hip extension moment. Of course, another perspective could be that the hip flexion moment derived under the device driving condition is too excessive to flex the hip joint angular displacement pattern continuously during the swing phase, in which case, the hip extension moment would have to be generated to compensate for excessive input joint moments, and, as a result, the selective activation of the GM, VL, and VM would play a counteraction role. ${ }^{(27)}$

\section{Conclusion}

We have constructed a gait training device that is based on master-slave systems and uses a hip joint angular controller. This device was able to reproduce a user's own gait motions; however, the patterns of hip joint moments and EMG activities in the lower limbs throughout one gait cycle were different from those that occur under the normal walking condition. Thus, when developing a gait training device to be used with the hip joint, the state of the intended user's movement disabilities should be carefully considered so that either a kinematics-, kinetics-, or EMG-based controller can be appropriately selected for the device.

\section{Acknowledgements}

This study was supported in part by KAKENHI (17700467), MIKIYA Science and Technology Foundation, Soft-Path Engineering Research Center (SPERC), and Faculty of Engineering, Iwate University; the pneumatic control equipment was provided by SMC Corp.

\section{References}

1 Y. Kita: Homepage of StrokeProject, http://www.stroke-project.com (accessed October 4, 2015) (in Japanese).

2 Nikkei BP: Homepage of Nikkei Medical Online, http://medical.nikkeibp.co.jp/leaf/mem/pub/series/ cvd/201307/531535.html (accessed October 4, 2015) (in Japanese).

3 A. Wernig, S. Muller, A. Nanassy, and E. Cagol: Eur. J. Neurosci. 7 (1995) 823.

4 V. Dietz: Nat. Rev. Neurosci. 3 (2002) 781.

5 N. Kawashima, D. Nozaki, M. O. Abe, M. Akai, and K. Nakazawa: J. Neurophysiol. 93 (2005) 777.

6 A. Takahashi: Jap. J. Phys. Ther. 42 (2008) 259.

7 S. C. Cramer, E. L. Orr, M. J. Cohen, and M. G. Lacourse: Exp. Brain Res. 177 (2007) 233.

8 L. Marchal-Crespo and D. J. Reinkensmeyer: J. Neuroeng. Rehabil. 6 (2009) 20.

9 G. Colombo, M. Joerg, R. Schreier, and V. Dietz: J. Rehabil. Res. Dev. 37 (2000) 693.

10 S. Hesse, C. Werner, and A. Bardeleben: Spinal cord 42 (2004) 346.

11 T. Miyoshi, K. Hiramatsu, S. I. Yamamoto, K. Nakazawa, and M. Akai: Disabil. Rehabil. 30 (2008) 81.

12 B. Tondu and P. Lopez: IEEE Contr. Sys. Magazine 20 (2000) 15.

13 K. C. Wickramatunge and T. Leephakpreeda: Int. J. Eng. Sci. 48 (2010) 188.

14 D. A. Winter: Biomechanics and motor control of human movement (2nd ed) (Wiley-Interscience, New York, 1990) Chap. 3.

15 T. Miyoshi, T. Shirota, S. I. Yamamoto, K. Nakazawa, and M. Akai: Disabil. Rehabil. 26 (2004) 724.

16 N. A. Alibeji, N. A. Kirsch, and N. Sharma: Front. Bioeng. Biotechnol. 2015 Dec 21;3:203. doi: $10.3389 /$ fbioe. 2015.00203

17 J. R. Koller, D. A. Jacobs, D. P. Ferris, and C. D. Remy: J. Neuroeng. Rehabil. 2015 Nov 4;12:97. doi: 10.1186/s12984-015-0086-5 
18 H. Sadeghi, S. Sadeghi, F. Prince, P. Allard, H. Labelle, and C. L. Vaughan: Clin. Biomech. 16 (2001) 688.

19 T. Miyoshi, T. Shirota, S. I. Yamamoto, K. Nakazawa, and M. Akai: Clin. Biomech. 20 (2005) 194.

20 A. A. Ebid, S. M. El-Shamy, and A. H. Draz: Burns. 2014 Feb;40(1):97-105. doi: 10.1016/j.burns.2013.05.022

21 H. Kawamura, S. Fuchioka, S. Inoue, S. Kuratsu, H. Yoshikawa, K. Katou, and A. Uchida: Scand. J. Rehabil. Med. 31 (1999) 77.

22 T. Miyoshi, K. Asaishi, T. Nakamura, M. Seki, T. Iguchi, K. Ichiryu, and M. Takagi: J Musculoskeletal Med. 26 (2015) 311.

23 A. L. Hof: J. Biomech. 34 (2001) 1085.

24 D. Nozaki, K. Nakazawa, and M. Akai: J. Neurophysiol. 93 (2005) 2614.

25 T. Miyoshi, Y. Takahashi, H. K. Lee, M. Yamaguchi, and T. Komeda: Proceedings of 2009 International Conference on Rehabilitation Robotics (IEEE, 2009) p. 442.

26 T. Miyoshi, M. Takagi, H. Saito, A. Yimit, and Y. Hagihara: New Trends on System Sciences and Engineering (IOS Press, Amsterdam, 2015) pp. 367-377.

27 T. Miyoshi, K. Nakazawa, M. Tanizaki, T. Sato, and M. Akai: Gait Posture 24 (2006) 94. 